\title{
Recognizing and Interpreting Sign Language Gesture for Human Robot Interaction
}

\author{
Shekhar Singh \\ Assistant Professor \\ CSE, Department \\ PIET, Samalkha, Panipat, India
}

\author{
Akshat Jain \\ Assistant Professor \\ CSE, Department \\ PIET, Samalkha, Panipat, India
}

\author{
Deepak Kumar \\ Assistant Professor \\ CSE, Department \\ PIET, Samalkha, Panipat, India
}

\begin{abstract}
Visual interpretation of sign language gesture can be useful in accomplishing natural human robot interaction. This paper describes a sign language gesture based recognition, interpreting and imitation learning system using Indian Sign Language for performing Human Robot Interaction in real time. It permits us to construct a convenient sign language gesture based communication with humanoid robot. The classification, recognition, learning, interpretation process is carried out by extracting the features from Indian sign language (ISL) gestures. Chain code and fisher score is considered as a feature vector for classification and recognition process. It is to be done by the two statistical approaches namely known as Hidden Markov Model (HMM) technique and feed forward back propagation neural network (FNN) in order to achieve satisfactory recognition accuracy. The sensitivity, specificity and accuracy were found to be equal $98.60 \%, 97.64 \%$ and $97.52 \%$ respectively. It can be concluded that FNN gives fast and accurate recognition and it works as promising tool for recognition and interpretation of sign language gesture for human computer interaction. The overall accuracy of recognition and interpretation of the proposed system is $95.34 \%$. Thus, this approach is suitable for automated real time human computer interaction tool.
\end{abstract}

\section{General Terms}

Human Robot Interaction; Gesture, Indian Sign Language; Vector Quantization and LBG algorithm; Hidden Markov Model; Neural Network.

\section{Keywords}

HRI, ISL, CLAHE, Chain Code, HMM, Fisher Score, FNN, Gesture recognizing, Gesture Interpretation

\section{INTRODUCTION}

The growing commitment of the society in reducing the barriers to persons with disabilities, added to the advances of the computers and form recognition methods, has motivated the development of the present system that recognized, learn and interpret ate the Indian sign language gesture .Sign language gesture based recognition, learning and interpretation is one of the most promising areas in research appealing its huge applications [28]. An artificial intelligent framework is being built with sign language gesture in order to accelerate Human Robot Interaction accurately. We have chosen suitable recognition, learning, interpretation techniques for the establishment of gesture based communication with humanoid robot. According to the different researcher point of view, hidden markov model (HMM) is a very well acceptable method for gesture recognition. But, we find that it takes more time to recognize the sign language gesture. For this reason we are trying to find a different method which gives same accuracy to recognize, learn and interpret ate sign language gestures but within a very short period of time. Indian Sign Language (ISL) has its own grammatical and syntactical meaning in the linguistic form of signs. It implies a visual-spatial language which consists of hands, arms, and facial expression and head or body postures in such a manner that linguistic information could be provided significantly. The construction of Indian sign language (ISL) gesture $[1,28]$ can be defined by several parameters like shape of the hand, location of the hand movements in a straight or circular way, orientation of the hand, facial expressions, body or head posture and eye gaze. The sign language gestures primitives are being captured by Indian Sign Language symbols. It imparts a challenge in terms of its complex symbolic gestural representation and proper linguistic understanding. It would be used as a helping agent for interpreting the knowledge among hearing impaired people in their own community. It will increase their strength of conversation in an extreme extent. This paper tries to introduce the strategy for dealing with the dynamic hand gesture recognition, learning and interpretation with reduction of time which will really be helpful for designing a concrete human robot interaction (HRI) system. It incorporates the challenges to extract and interpret ate the dynamic feature from the continuous signals.

The current prototype strongly supports the mimicry on humanoid robot in real time. The further expansion on recognition, learning and interpretation includes the translation system between verbal expression and sign language. This system would be useful for hearing impaired people for exchanging information among them through the human robot interaction (HRI) approach. This demands a real time gesture recognition system by translating sign language. The unique and novel techniques using neural network and HMM have been applied separately in our current system to recognize the Indian sign language (ISL) gestures and generate mimicry by the humanoid robot accordingly. Then we try to identify a suitable recognition, learning, interpretations system according to the time taken for recognizing learn, and interpreted the sign language gesture. The used procedure in this automatic recognition is based in the adequate modeled of the hand sign by Neural Network. For it, it has been realized the calculation of Fisher score, which is extracted of HMM [28, 31]. This HMM is fed with the chain code, determined from the band sign image. For this process, the first step has been arranged the capture of samples. It is necessary to create a database with hand sign pictures of the sign text of numerous persons, being the ideal infinite. In our case, it was enclosed for sixty different people (50 hand signs each one). Subsequently, it has been applied a image preprocessing, transforming the color image in a white and black one, with more reduced size, which defines the 
hand outline. With this outline, we are going to extract a series of parameter, associated in a vector that defines the contour of the hand sign with chain code [29, 32], and it is classified and parameterized by HMM. From these HMM, some parameters are going to extract, based on the emission probability of the vectors, which are going to determine the score Fisher [28, 32], for classifying it with Neural Network $[33,34,39,40]$. This system will he supervised with a training process, in which is learned to differentiate some hand signs of other; and a other test process, where the models will be verified. This process is resumed in the following figure 1 . In the second section the creation of the database. In the third, the applied image processing. Subsequently, the calculation of Fisher score by HMM. In the fifth section the neural network classifier. The sixth sections are all the realized experiments and finally the conclusions and references. Figure 1 shows the proposed system for recognizing and interpretation of sign language gesture for human robot interaction.

\section{INDIAN SIGN LNGUAGE GESTURE ACQUISITION SYSTEM}

The prime focus on recording Indian sign language gesture is to create a repository with dynamic ISL video (sequence of images) gestures from different kinds of Indian sign language class/word dictionary. Preliminarily, Indian sign language dynamic gestures have been recorded with fixed frame rate per second (10 fps) and with the fixed location of the camera from the object. The samples of Indian sign language gestures are to be used during the classification, learning and interpretation process. All the Indian sign language gestures include various kinds of hand motions. A capturing device SONY handy cam with 8 mega pixel resolutions is used for capturing videos of several Indian sign language gestures. One elementary approach for image processing tends to background uniformity where a dark background is chosen for dealing with gray scale images effectively.

To have a controlled environment, the background uniformity has been kept while recording the videos in real time. This will reduce the computational complexity during background removal and increase recognition and interpretation accuracy in real time [15]. A single gesture video has been restricted to 20 frames. This is done by selecting 20 frames equally spaced in time from the original captured video. The background is chosen dark. Every ISL gesture implies some class or word which could be captured by waving both hands in a very appropriate manner [16]. For the enhancement of preprocessing Indian sign language gestures need fast and accurate movements of hands. Several operations have been accomplished in all the Indian sign language (ISL) videos before the classification process.

\section{PREPROCESSING AND FEATURE EXTRACTION}

Primarily, all the Indian sign language videos are split up into sequences of image frames (RGB). The frames are converted into grayscale images and the background is subtracted in order to reduce computational complexity. In this process are going to take the color images for transforming to binary images of hand sign shape (white and black) with a fixed height of 400 pixels, conserving the relation with respect the width. The following steps are;

1. Filter RGB image for eliminating the noise, hue, saturation effects.
2. Convert RGB image in YCBCR color image by eliminating the hue and saturation [40]

3. Convert YCBCR image in grayscale by eliminating the hue and saturation [40].

4. Filter grayscale image for eliminating the noise, hue and saturation.

5. Enhance contrast using histogram equalization [29]. To realize this process, it is equalized the histogram of the different levels of gray, under a lineal function, but without affecting to the darkest points, only for the clearest parts (the hand sign), marking differences among the shadow of the hand and the background; and the own hand.

6. Take the frame out to eliminate some border effects.

7. Convert image to binary image by Thresholding. The Thresholding is computed by means of Otsu's method, which chooses the threshold to minimize the interclass variance of the Thresholding black and white pixels. With this step is finished for determining the hand as an object.

8. Morphologic operators [30]. It is applied the dilatation operations in a first place, and after the erosion, as effect to recover the conditions of size of the image. The elimination of the noise is other desired effect. The dilatation is going to unite the holes that can remain in the line of the contour. Subsequently the hand is filled as object, and finally recovers the original dimension, by means of the erosion, that is able to eliminate the possible noises happened by the own photo.

9. Reduction. The size of image is reduced in one quarter, for reducing the size of the final vector.

10. Calculation of the contour $[31,32]$. It is calculated the hand contour, which determines the hand sign on the background of the image, with the particularity that the connection among pixels is alone one pixel, with a connectivity among neighbors of 8 , that is chain code.

11. Adjustment of the wrist. The image is trimmed slightly with the intention to determine the side of the one that the hand sign arises. Adjustment of high of the image. Finally it is fixed the value of the height, maintaining the proportionality with regard to the width. In this way the information is not loosen, indistinctly that the hand sign is horizontal, vertical up or vertical downward.

Figure 1 shows the process of image preprocessing and feature extraction step of proposed system. Figure 1 shows the proposed system for recognizing and interpretation of sign language gesture for human robot interaction. The feature extraction for hand gesture recognition is done using chain code and fisher score [17]. It provides tremendous flexibility towards scene illumination invariance property. The classification and interpretation policy includes robustness in changing the illumination conditions. The fisher score of dynamic gestures forms the feature vector based on the image feature. HMM and neural network are very robust and efficient algorithm which would be used to classify ISL dynamic gestures based on the pattern classification and recognizing technique. The algorithm is constituted with the chain code and fisher score of the local orientation of edges in an image. The chain code and fisher score will be treated as feature vector for motion classification of Indian sign language gestures. The algorithm is very fast and strong to compute the feature vectors of the sequence of images. Therefore, the calculation of direction of edges can be performed in real time applications. It offers advantage to scene illumination changes and even light condition changes. The edges of the sequences of images would be still same [18]. All the Indian sign language gestures have been captured 
in different lighting conditions. Another advantage of chain code and fisher score refers to the translation invariant property. It demonstrates that the same frames at different position of gestures would produce the same feature vectors. It is being done to calculate the chain code and fisher score of the local orientations for all the frames of the moving gestures. Translation of the frame in the gesture does not change the chain code and fisher score. The overall algorithm $[37,38]$ has been described to evaluate the feature vector for recognition, learning and interpretation system.

\section{ISL RECOGNITION AND INTERPRETATION TECHNIQUE}

The classification and interpretation process is done by two statistical techniques: HMM technique and neural network. Hidden Markov Model technique is followed by vector quantization technique with Linde Buzo Gray algorithm [38]. The HMM explains the construction of model which is needed to generate the observation sequences. Here we use a left-right HMM. The details of HMM is defined in the following manner [37]: In our research work we have generated 10 different HMM models for 21 different ISL gestures for classification and interpretation. Figure 1 shows the process of image processing, chain code calculation, fisher scores calculation, HMM, quantization, learning, recognizing and interpretation of sign language gesture.

\subsection{Fisher Score calculation}

Once it is obtained all the outline images of the hand signs, it is realized the calculation of Fisher score. This process comprises in three steps;

a. Extraction of parameter from outline: chain code [29].

b. Creation HMM with chain code as input $[31,32]$.

c. Calculation of Fisher score from gradient of logarithm of the observation symbol probability distribution [28, 32]

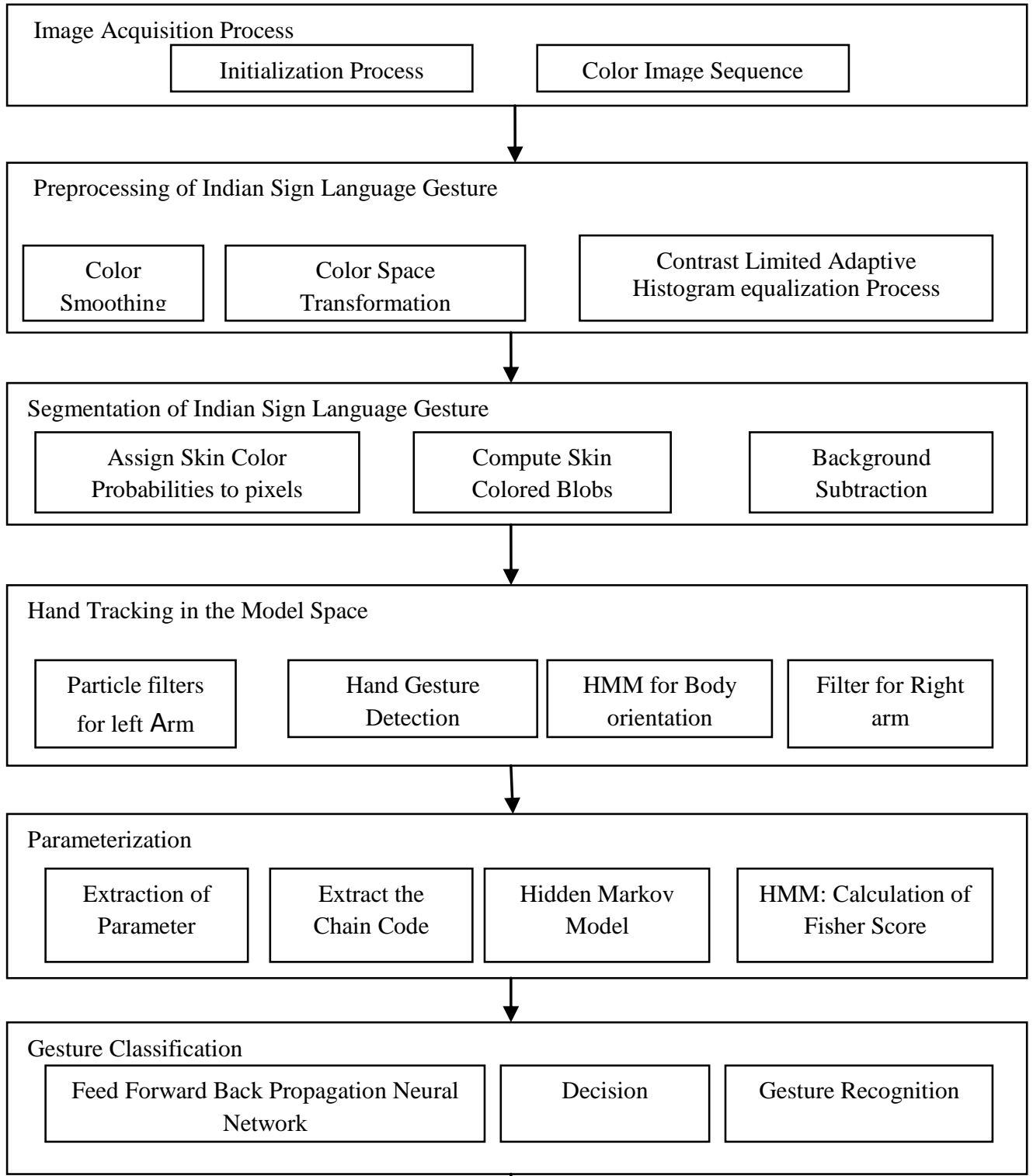

Fig 1: Block diagram of the proposed approach for hand tracking and gesture recognition. Processing is organized into six layers. 
This vector of the contour hand sign is obtained with the mask of the figure 2, observing the position of each pixel with their adjacent one. This vector is formed by numbers from the 1 to the 8 that describes the outline of the hand sign. The information that is extracted, describes the sequence of the hand, accompanied by temporary information, because all the hand signs are acquired in the same order and sense. This information is very important for the recognizer based on HMM, therefore utilizes this information to distinguish the different hand signs. It is fixed a criterion of start for the obtaining of this vector, verifying first if is a vertical, horizontal since down or vertical since up hand sign, for this is not rotation invariant. It is begun for seeking write pixels with the following order of priority: first, the first column of the left (horizontal hand sign), the last row (vertical since down) or the first row (vertical since up).

\begin{tabular}{|c|c|c|}
\hline 1 & 2 & 3 \\
\hline 8 & $X$ & 4 \\
\hline 7 & 6 & 5 \\
\hline
\end{tabular}

Fig 2: Mask of composition of the vector of the chain code

\section{2 Transformation of Parameter with HMM}

It is going to determine by the supervised classification of chain code using HMM, which is the maximum rate of success, for extrapolating the forward and backward parameter of HMM in the calculation of Fisher score. Therefore, the HMM employed is a Bakis, and is trained with the procedure of Baum-Welch, to maximize the probabilities of success [28]. Besides, 8 symbols by state have been utilized. The creation of the HMM models has two phases, the training and the test. Finally, the number of states (N) and the percentage of training samples have utilized like parameters to find the highest rate of success.

\subsection{Fisher Score}

Finally, it is proposed the transformation that provides the HMM probabilities relating to the approach of the Fisher score [28, 32]. With this goal, it intends to unite the probability given by the HMM to the given discrimination of the neural network, whose tie of union is this Fisher score. This score calculates the gradient with respect to the parameters of HMM, in particular, on the probabilities of emission of a vector of data $\mathrm{x}$, while it is found in a certain state $\mathrm{q} \in\{1, \ldots \ldots, \mathrm{N}\}$, given by the matrix of symbol probability in state $\mathrm{q}\left(\mathrm{b}_{\mathrm{q}}(\mathrm{x})\right)$, just as it is indicated in the following equation ;

$\mathrm{P}(\mathrm{x} / \mathrm{q}, \lambda)=\mathrm{b}_{\mathrm{q}}(\mathrm{x})$

If it is realized the derivate of the logarithm of the above probability, with the purpose to calculate its gradient, it is obtained the kernel of Fisher, whose expression comes given by;

$\partial \log \mathrm{p}(\mathrm{x} / \mathrm{q}, \lambda) / \partial \mathrm{p}(\mathrm{x}, \mathrm{q})=\zeta(\mathrm{x}, \mathrm{q}) / \mathrm{b}_{\mathrm{q}}(\mathrm{x})-\zeta(\mathrm{q})$

Where in $[28,31,32]$, it has been found the approximations and the calculation of above equation. Besides, $\zeta(\mathrm{x}, \mathrm{q})$ represents the number of times, that is localized in a state $\mathrm{q}$, during the generation of a sequence, emitting a certain symbol $\mathrm{x}[28,31] . \zeta(q)$ represents the number of times that has been in q during the process of generation of the sequence [28, 31]. These values are obtained directly and of form effective, from the forward backward algorithm, applied to the HMM [28, 31]. The application of this score $\left(\mathrm{U}_{\mathrm{x}}\right)$ to the neural network, comes given by the expression of the equation 2, utilizing the techniques of the natural gradient, from the following equation [1];

$\mathrm{U}_{\mathrm{x}}=\nabla_{(\mathrm{x}, \mathrm{q})} \log \mathrm{p}(\mathrm{x} / \mathrm{q}, \lambda)$

(Eq. 3)

Where $U_{x}$ define the direction of maximum slope of the logarithm of the probability to have a certain symbol in a state.

\subsection{Feed forward back propagation Technique}

The objective of this study is to classifying Fisher kernel data of sign language latter symbol using feed forward back propagation neural network and Levenberg-Marquardt (LM) as the training algorithm. LM algorithm has been used in this study due to the reason that the training process converges quickly as the solution is approached. For this study, sigmoid, hyperbolic tangent functions are applied in the learning process. Feed forward back propagation neural network use to classify sign language gesture according to fisher score characteristic [33, 34, 39, and 40]. Feed forward back propagation neural network is created by generalizing the gradient descent with momentum weight and bias learning rule to multiple layer networks and nonlinear differentiable transfer functions. Input vectors and the corresponding target vectors are used to train feed forward back propagation neural network. Neural network train until it can classify the defined pattern. The training algorithms use the gradient of the performance function to determine how to adjust the weights to minimize performance. The gradient is determined using a technique called back propagation, which involves performing computations backwards through the network. The back propagation computation is derived using the chain rule of calculus. In addition, the transfer functions of hidden and output layers are tan-sigmoid and tan-sigmoid, respectively.

\section{Training and Testing:}

The proposed network was trained with fisher score data cases. When the training process is completed for the training data, the last weights of the network were saved to be ready for the testing procedure. The time needed to train the training datasets was approximately 4.60 second. The testing process is done for 60 cases. These 60 cases are fed to the proposed network and their output is recorded.

\section{VECTOR QUANTIZATION TECHNIQUE}

A discrete HMM is taken into consideration for recognition process of Indian sign language gestures. The feature vector of chain code needs to be converted into a finite set of symbols from a codebook. The VQ technique plays a reference role in HMM based approach in order to convert continuous Indian sign language (ISL) gestural signals into a discrete sequence of symbols for discrete HMM. The VQ concept is entirely determined by a codeword which is composed by fixed prototype vectors. Fig 3 shows that the process of quantization has been divided into two parts. The first part is having the ability to produce a codebook and the 
second part attempts to update the codeword followed by training of all the vectors according to their finite vectors. Its strength lies in reducing the data redundancy and the distortion created among the quantized data and the original data. It is essentially required to propose a VQ method which would be genuinely used to minimize this distortion measure. In order to compute the minimum average distortion measure for a set of vectors an iterative algorithm is proposed by Linde, Buzo and Gray [22] which is known as LBG vector quantization designing algorithm. The algorithm illustrates the generation of optimal codebook (in our case codebook size is 16) for isolated ISL gestures.

\section{RECOGNIZING AND INTERPRETATION BY HUMANOID \\ ROBOT}

Fig 3 shows that the process of quantization, learning, recognizing and interpretation of sign language gesture. An underlying concept of learning gestures has been introduced for humanoid robot in order to perform several tasks eventually [27]. An integration of humanoid robot with Indian sign language gestures encounters an elegant way of communication through mimicry. The real time robotics simulation software, WEBOTS is adopted to generate HOAP2 actions accurately. The way of learning process marks an intelligent behavior of HOAP-2 which sustains its learning capability in any type of environment. The learning process is dealt with the HOAP-2 robot controller which has been built intelligently. It is used to invoke Comma Separated Value (CSV) file in order to perform that gestures in real time. All the classified gestures bring out some useful information about all the joints of upper body of the humanoid robot.

\subsection{Learning ISL gesture using HMM with vector quantization techniques}

In order to learn the ISL gestures by humanoid robot the preprocessing technique is essentially needed for this purpose. It employs the following steps.

* Capture the ISL gesture as an input gesture.

* Apply an algorithm for extracting orientation histogram to construct feature vector.

* From the feature vector of each gesture an initial codebook is to be generated. Then apply LBG algorithm to generate an optimized codebook.

* Each row corresponds to a number of the codeword which helps to form a quantized vector used by $\mathrm{hmm}$ algorithm.

In this preprocessing step we have generated 30 symbol sequences for each ISL gesture as each gesture is captured with equal number of frames. Next stage implies to train each gesture using Hidden Markov Model where parameters of the HMM can be determined efficiently. We calculated the transition probability and emission probability of HMM by the known states and known sequences. The training algorithm for each gesture measures the accurate transition and emission probability which are used for finding out the most probable sequence.

It has been assumed 5 hidden states for each ISL gesture and made the state sequences in the distribution of 1 to 5 with total number sequence matches the codebook size. The observation sequence for each state has been represented by row vector with the calculated codebook of all the training samples. Each observation sequence for each training gesture corresponds to single row vector. Then apply algorithm for the estimation of transition and emission probability of each gesture model and preserve it for recognition purpose with unknown gesture. Every HMM model is uniquely attached with each gesture which is trained with different samples of each ISL gesture. This trained model is extensively used for recognition of new gesture. This new gesture would be tested through all the trained HMM model. The new gesture is to be declared as classified when it provided a maximum likelihood state with a trained gesture. Compute the percentage of each probable state for all the training samples which agrees with the likely state sequences of test gesture. The gesture is declared as classified with maximum percentage. Fig 3 shows the process of quantization, learning, recognizing and interpretation of sign language gesture using HMM and neural network.

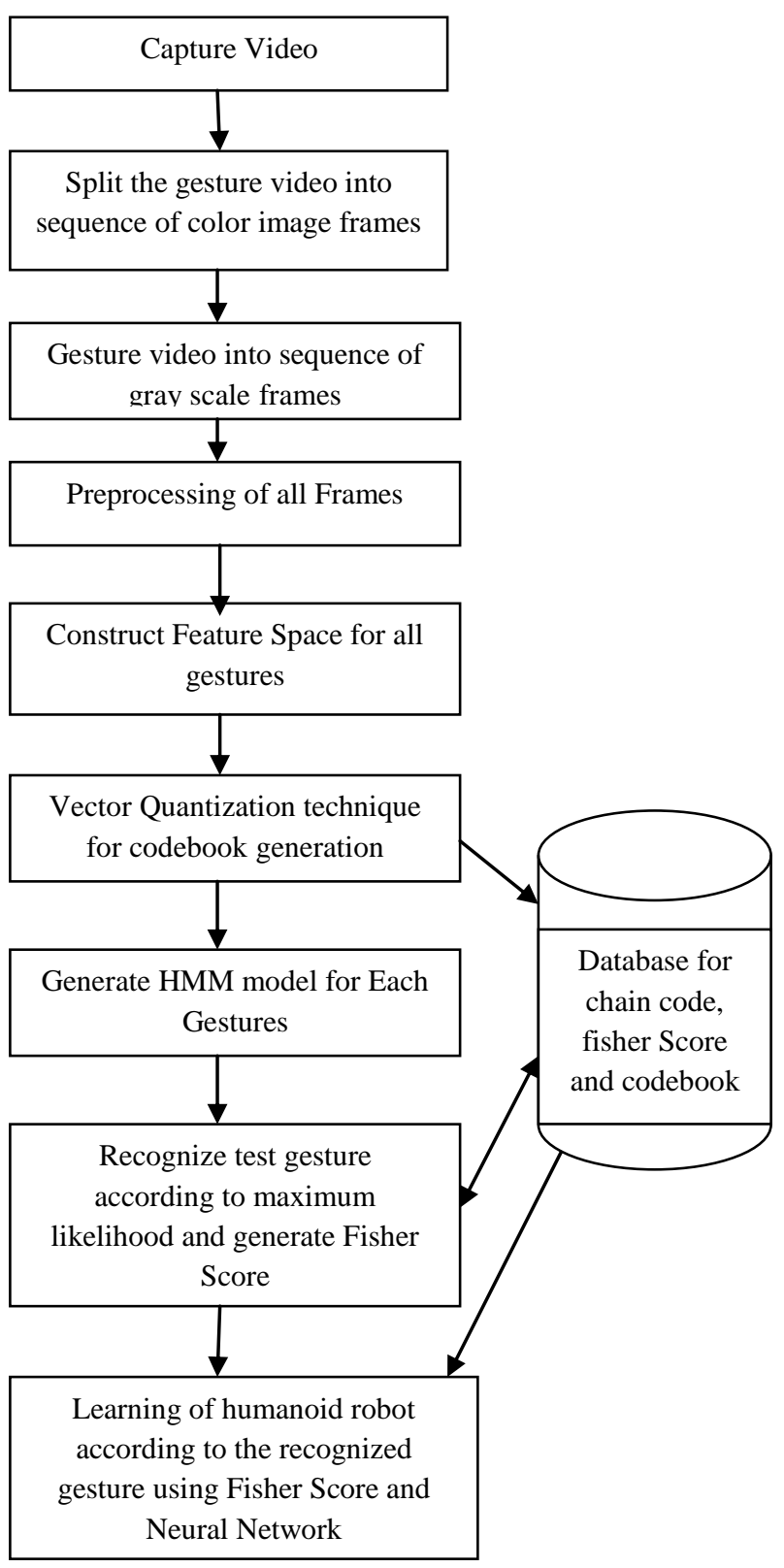

Fig 3: Learning of ISL gesture using HMM, Fisher Score and Neural Network technique 


\subsection{Learning ISL gesture using Neural Network}

The objective of this study is to classifying Fisher kernel data of sign language gesture using feed forward back propagation neural network and Levenberg-Marquardt (LM) as the training algorithm. Feed forward back propagation neural network use to classify sign language gesture according to fisher score characteristic [33, 34, 39, and 40]. Fig 3 shows the process of quantization, learning, recognizing and interpretation of sign language gesture using neural network.

\section{RESULT ANALYSIS}

Only 21 ISL gestures are selected for our work. In our work the same gesture is performed by the ten different persons. During acquisition of ISL video gestures we have kept same number of frames for each gesture. It makes the recognition process easier but it reduces the freedom of doing the gesture. In the HMM classification process each model is tested by Viterbi algorithm, where same number of frames are required to get percentage of the matching gesture. We have taken 10 samples of each gesture for training and 10 separate samples for each gesture for testing. We have generated probable path from the trained hidden model for each training samples of each gesture using viterbi algorithm. The same process is also applied for test samples of each gesture. In the recognition phase of known gestures, we have taken test sample one by one from the test gestures and have compared that sample with all the samples of each trained gestures iteratively. It produces the recognition percentage of that particular sample into the entire training samples. In that process we have created separate training set and test set with most probable paths. If a particular gesture is matched with $60 \%$ and above according to the maximum likelihood state with one of the training samples of that gesture then it is considered as classified gesture. We have achieved up to $98 \%$ recognition accuracy with both the techniques. The total prototype is tested and simulated on Intel Core 2 Duo system with Mat lab coding. Each gesture for mimicry generation needs a special care of its dedicated joints which are responsible to perform that gesture. The experiments have been realized with independent samples for training and for test. These have been repeated in five times to take averaged values, expressed them by their average and their variance.

Table 1: Rate of success of the HMM in function of the percentage of samples for training and of the number of states

\begin{tabular}{|l|l|l|l|l|l|}
\hline \multirow{2}{*}{$\begin{array}{l}\text { Samples } \\
\text { Trainin } \\
\text { g }\end{array}$} & \multicolumn{4}{|l|}{ Number of state } \\
\cline { 2 - 6 } & $\mathbf{2 0}$ & $\mathbf{4 5}$ & $\mathbf{4 5}$ & $\mathbf{6 5}$ & $\mathbf{1 0 0}$ \\
\hline $20 \%$ & 75.66 & 86.60 & 87.74 & 85.38 & 64.40 \\
& $\%$ & $\%$ & $\%$ & $\%$ & $\%$ \\
& \pm 22.82 & \pm 3.33 & \pm 2.47 & \pm 7.67 & \pm 45.65 \\
\hline $40 \%$ & 78.20 & 88.70 & 88.58 & 92.10 & 73.20 \\
& $\%$ & $\%$ & $\%$ & $\%$ & $\%$ \\
& \pm 12.83 & \pm 3.50 & \pm 7.24 & \pm 1.82 & \pm 26.50 \\
\hline $60 \%$ & 76.78 & 91.90 & 87.30 & 87.98 & 68.10 \\
& $\%$ & $\%$ & $\%$ & $\%$ & $\%$ \\
& \pm 11.20 & \pm 2.95 & \pm 3.90 & \pm 3.10 & \pm 25.87 \\
\hline $80 \%$ & 77.31 & 92.75 & 93.20 & 92.50 & 75.65 \\
& $\%$ & $\%$ & $\%$ & $\%$ & $\%$ \\
& \pm 31.42 & \pm 14.19 & \pm 8.30 & \pm 14.15 & \pm 16.80 \\
\hline
\end{tabular}

The execution of the same have been done sequential mode, first in the HMM to achieve its maximum success, varying the number of states and the percentage of samples in the training.
The experimental results when varying the number of states and states of HMM are shown in table 1. As a result, the recognition accuracy was improved by increasing the number of states and the highest recognition accuracy was obtained in 65 states. Although the recognition accuracy was hardly improved when the number of samples increased, the highest recognition accuracy was obtained in $80 \%$ samples. As a result, recognition accuracy was $94.50 \%$ by using only the position of hands. The results are show in table1. Of the above table is deduced that the best percentage of training is fur $80 \%$ and 65 states, with a rate of $94.50 \%$, presenting a small variance. Of this model is going to generate the kernel of Fisher, and it is going to apply to the neural network. It is observed as for the neural networks are presented better results, arriving to an average rate of $95.34 \%$, with the smallest variance. The overall accuracy of Indian sign language gesture recognition in the training, validation and testing mode are $98.60,97.64$ and $97.52 \%$.

\section{CONCLUSION}

In this work we have used Indian sign language gesture as a communicating agent between human and robot interaction. This is our first step to design a prototype of vision base human robot interaction (HRI) system for speech and hearing impaired persons. They can use the humanoid robot as his translator or as his helping agent where the persons could communicate with the robot through Indian sign language gesture. According to the observation we select neural network as a best recognition tool compatible with fisher score according to time taken and accuracy for recognizing gestures. However in the present work we are only implementing a mimicry action by the humanoid robot. We could in principle use these techniques for trying to recognize any other human gestures. However the complexity of classification will increase because of the ambiguity in normal human gestures. We have chosen ISL because of its rigid vocabulary which makes the classification simpler. The present work only recognizes a single gesture at a time. It will be challenging to recognize multiple gestures or sequence of gesture one after the other. The task in hand will be to separate out each gesture from the sequence of gestures and use our gesture recognition techniques as described in this paper. In this article a robust and novel automatic Indian sign language gesture recognition system has been presented. The overall accuracy of sign language recognition in the training, validation and testing mode are 98.60, 97.64 and $97.52 \%$. We are concluding that that the proposed system gives fast and accurate Indian sign language gesture recognition. Given the encouraging test results, we are confident that an automatic sign language gesture recognition system can be developed.

\section{REFERENCES}

[1] Zhang, J., Zhao, M.: A vision-based gesture recognition system for human-robot interaction. Robotics and Biomimetics (ROBIO), 2009 IEEE International Conference on, vol., no., pp.2096-2101, 19-23 Dec. (2009). doi: 10.1109/ROBIO.2009.5420512

[2] Calinon, S., Guenter, F., Billard, A.: On Learning, Representing and Generalizing a Task in a Humanoid Robot. IEEE Trans. on Systems, Man and Cybernetics, Part B, Vol. 37, No. 2, pp. 286-298 (2007). doi: 10.1109/TSMCB.2006.886952 
[3] Calinon, S., Guenter, F., Billard, A.: Goal-Directed Imitation in a Humanoid Robot. International Conference on Robotics and Automation (ICRA), pp. 299-304 (2005).

[4] Pantic, M., Rothkrantz, L. J. M.: Toward an affectsensitive multimodal human-computer interaction. IEEE, vol.91, no.9, pp. 1370- 1390, Sept. (2003).

[5] Bhuyan, M. K., Ghoah, D., Bora, P. K.: A Framework for Hand Gesture Recognition with Applications to Sign Language. India Conference, 2006 Annual IEEE, PP. 16, Sept. (2006). doi: 10.1109/INDCON.2006.302823

[6] Prasad, J. S., Nandi, G. C.: Clustering Method Evaluation for Hidden Markov Model Based Real-Time Gesture Recognition. Advances in Recent Technologies in Communication and Computing, ARTCom '09, pp. 419-423, 27-28Oct. (2009)

[7] Lee, H. J., Chung, J. H.: Hand gesture recognition using orientation histogram. TENCON 99. Proceedings of the IEEE Region 10 Conference, vol.2, no., pp.1355-1358 vol.2, Dec. (1999). doi: 10.1109/TENCON.1999.818681

[8] Freeman, W. T., Roth, M.: Orientation histograms for hand gesture recognition. Intl. Workshop on Automatic Face- and Gesture- Recognition, IEEE Computer Society, Zurich, Switzerland, pp.296-301, June (1995). MERL-TR94-03.

[9] Nandy, A., Prasad, J. S., Chakraborty, P., Nandi, G. C., Mondal, S.: Classification of Indian Sign Language In Real Time. International Journal on Computer Engineering and Information Technology (IJCEIT), Vol. 10, No. 15, pp. 52-57, Feb. (2010).

[10] Nandy, A., Prasad, J. S., Mondal, S., Chakraborty, P., Nandi, G. C.: Recognition of Isolated Indian Sign Language gesture in Real Time. BAIP 2010, Springer LNCS-CCIS, Vol. 70, pp. 102-107, March (2010). doi: 10.1007/978-3-642-12214-9_18.

[11] Dasgupta, T., Shukla, S., Kumar, S., Diwakar, S., Basu, A,: A Multilingual Multimedia Indian Sign Language Dictionary Tool. The 6'Th Workshop on Asian Language Resources, pp. 57-64 (2008).

[12] Kim, J., Thang, N. D., Kim, T.: 3-D hand motion tracking and gesture recognition using a data glove. Industrial Electronics, 2009. ISIE 2009. IEEE International Symposium on, vol., no., pp.1013-1018, 5-8 July (2009). doi: 10.1109/ISIE.2009.5221998

[13] Jiangqin, W., Wen, G., Yibo, S., Wei, L., Bo, P.: A simple sign language recognition system based on data glove. Signal Processing Proceedings, 1998. ICSP '98. 1998 Fourth International Conference on, vol.2, no., $\begin{array}{lll}\text { pp.1257-1260 vol.2 (1998). doi: } & \end{array}$ 10.1109/ICOSP.1998.770847

[14] Ishikawa, M., Matsumura, H.: Recognition of a handgesture based on self-organization using a DataGlove. Neural Information Processing, 1999. Proceedings. ICONIP '99. 6th International Conference on, vol.2, no., pp.739-745 vol.2 (1999). doi: 10.1109/ICONIP.1999.845688

[15] Swee, T. T., Ariff, A. K., Salleh, S. H., Seng, S. K., Huat, L. S.: Wireless data gloves Malay sign language recognition system. Information, Communications \& Signal Processing, 2007 6th International Conference on, vol., no., pp.1-4, 10-13 Dec. (2007). doi: 10.1109/ICICS.2007.4449599

[16] Liang, R. H., Ouhyoung, M.: A real-time continuous gesture recognition system for sign language. Automatic Face and Gesture Recognition, 1998. Proceedings. Third IEEE International Conference on, vol., no., pp.558-567, 14-16 Apr (1998).

[17] Won, D., Lee, H. G., Kim, J. Y., Choi, M., Kang, M. S.: Development of a wearable input device based on human hand-motions recognition. Intelligent Robots and Systems, 2004. (IROS 2004). Proceedings. 2004 IEEE/RSJ International Conference on, vol.2, no., pp 1636- 1641 vol.2, 28 Sept.-2 Oct. (2004). doi: 10.1109/IROS.2004.1389630

[18] Kuzmanic, A., Zanchi, V.: Hand shape classification using DTW and LCSS as similarity measures for visionbased gesture recognition system. EUROCON, 2007. The International Conference on "Computer as a Tool", vol., no., pp.264-269, 9-12 Sept. (2007). doi: 10.1109/EURCON.2007.4400350

[19] Hienz, H., Grobel, K., Offner, G.: Real-time hand-arm motion analysis using a single video camera. Automatic Face and Gesture Recognition, 1996., Proceedings of the Second International Conference on , vol., no., pp.323327, 14-16 Oct. (1996).

[20] Hasanuzzaman, M., Ampornaramveth, V., Zhang, T., Bhuiyan, M. A., Shirai, Y., Ueno, H.: Real-time Visionbased Gesture Recognition for Human Robot Interaction. Robotics and Biomimetics, 2004. ROBIO 2004. IEEE International Conference on, vol., no., pp.413-418, 22-26 Aug. (2004). doi: 10.1109/ROBIO.2004.1521814.

[21] Rabiner, L. R.: A tutorial on hidden Markov models and selected applications in speech recognition. IEEE, vol.77, no.2, pp.257-286, Feb. (1989). doi: 10.1109/5.18626.

[22] Vector Quantization Technique and LBG Algorithm. www.cs.ucf.edu/courses/cap5015/vector.ppt

[23] Michailovich, O., Rathi, Y., Tannenbaum, A.: Image Segmentation Using Active Contours Driven by the Bhattacharyya Gradient Flow. IEEE Transactions on Image Processing, vol.16, no.11, pp.2787-2801, Nov. (2007). doi: 10.1109/TIP.2007.908073

[24] Kailath, T.: The Divergence and Bhattacharyya Distance Measures in Signal Selection. IEEE Transactions on Communication Technology, vol.15, no.1, pp.52-60, Feb. (1967).

[25] Nayak, S., Sarkar, S., Loeding, B.: Distribution-Based Dimensionality Reduction Applied to Articulated Motion Recognition. Pattern Analysis and Machine Intelligence, IEEE Transactions on, vol.31, no.5, pp.795-810, May (2009). doi: 10.1109/TPAMI.2008.80.

[26] Nandy, A., Mondal, S., Prasad, J. S., Chakraborty, P., Nandi, G. C.: Recognizing \& interpreting Indian Sign Language gesture for Human Robot Interaction. Computer and Communication Technology (ICCCT), 2010 International Conference on , vol., no., pp. 712-717, 17-19 Sept. (2010). doi: 10.1109/ICCCT.2010.5640434.

[27] Mitra, S., Acharya, T.: Gesture Recognition: A Survey. IEEE Transactions on Systems, Man, and Cybernetics, Part C: Applications and Reviews, vol.37, no.3, pp.311324, May (2007). doi: 10.1109/TSMCC.2007.893280. 
[28] Lawence R. Rabiner, "A tutorial on Hidden Markov models and Selected Applications in Speech Recognition", in Proceedings of the IEEE, vol. 77, no. 2,IEEE, pp. 257-286, 1989.

[29] L. O'Gorman and R. Kasturi, Document Image AnalJsis, IEEE Computer Society Press, 1995.

[30] J. Serra, Image Analysis and Mathematical Morphology, Academic Press, 1982.

[31] C. Travieso, C. Morales, I. Alonso y M. Ferrer, "Handwritten digits parameterisation for HMM based recognition", Proceedings of the Image Processing and its Applications,vol.2, pp. 770-774, julio de 1999.

[32] E. Gomez, C.M. Travieso, J.C. Briceiio, M.A. Ferrer, "Biometric Identification Svstem by Lip Shape", in Proceeding of 361h International Carnahan Conference on Security Technology, Atlantic City, October 2002,pp. $39-42$.

[33] L. Fausett, "Fundamentals of Neural Networks, Architectures, Algorithms, and Applications", PrenticeHall, Inc. 1994, pp-304-315.

[34] K.Murakami, H.Taguchi: Gesture Recognition using Recurrent Neural Networks. In CHI '91 Conference Proceedings (pp. 237-242). ACM. 1991.
[35] Chang, J. Chen, W. Tai, and C. Han, -New Approach for Static Gesture Recognition", Journal of Information Science and Engineering22, 1047-1057, 2006.

[36] S. Naidoo, C. Omlin and M. Glaser, "Vision-Based Static Hand Gesture Recognition Using Support Vector Machines", 1998.pages 88 - 94.

[37] Vladimir I. Pavlovic, Rajeev Sharma, Thomas S Huang, - Visual Interpretation of Hand Gestures for HumanComputer Interaction: A review\| IEEE Transactions of pattern analysis and machine intelligence, Vole 19, NO 7, July 1997, 677 - 695

[38] Visual gesture recognition. In proceedings IEEE Visual Image Signal Process, by Davis \& Shah, 1994 vol.141, Issue: $2,101-106$.

[39] Hand gesture Recognition of Radial Basis Functions (RBF) Networks and Decision Trees - International Journal of Pattern Recognition and Artificial Intelligence. Volume: 11, Issue: 6(1997) pp. 845-850.

[40] Face detection in complicated backgrounds and different illumination conditions by using $\mathrm{YCbCr}$ color space and neural network Pattern Recognition Letters, Volume 28, and Issue 16, 1 December 2007, Pages 2190-2200. 